\title{
Growing Older Does Not Always Mean Moving Slower: Examining Aging and the Saccadic Motor System
}

\author{
Jay Pratt \\ Department of Psychology \\ University of Toronto, Ontario, Canada \\ Michael Dodd \\ Department of Psychology \\ University of British Columbia \\ Vancouver, Canada
}

\author{
Timothy Welsh \\ Faculty of Kinesiology \\ University of Calgary \\ Alberta, Canada
}

\begin{abstract}
Although humans typically move more slowly as they age, one exception may be the saccadic motor system. To fully determine whether the execution of saccades is affected by age, the authors examined detailed kinematics of vertical and horizontal saccades across a range of saccadic amplitudes $\left(4^{\circ}, 8^{\circ}\right.$, and $\left.12^{\circ}\right)$. Ten younger and 20 older adults participated in each experiment. Whereas in the 1st experiment, the authors assessed volitionally generated saccades, in the 2 nd experiment, they evaluated reflexively generated saccades. The results of those experiments showed that the saccadic motor system is relatively impervious to the effects of aging; in fact, the differences between vertical and horizontal saccades were more evident than were differences between saccades produced by younger and older adults. The authors discuss possible reasons for that relative resistance to aging.
\end{abstract}

Key words: aging, eye movements, kinematics, saccades

A s humans age, they undergo a complex constellation of changes in both physical and mental functioning. With regard to mental abilities, some cognitive functions show marked changes in age, whereas others remain relatively intact across the life span. Questions about exactly which functions show-and which do not show-the effects of aging and why those changes may or may not occur punctuate the cognitive-aging literature. Although the effects of age on mental capacity vary, it is widely accepted that physical abilities decline with increased age. That apparent fact of life can be seen in most professional sports; players in their mid- to late 30s are considered old and must use their "veteran savvy" to make up for their diminishing physical skills. Such age-related reductions in speed and force can be seen when one looks at athletic world records across age groups. In terms of speed, the current $100-\mathrm{m}$ men's sprint world record is $9.77 \mathrm{~s}$, whereas the world record for men aged $70-75$ is $32 \%$ slower at 12.91 s. For force, the women's discus world record is $76.8 \mathrm{~m}$, whereas the 70-75-year-old women's world record is more than $50 \%$ less at $31.29 \mathrm{~m}$. Most people do not need to look to professional athletes or world records to know the effects of aging on physical abilities - they simply are not capable of the physical acts they performed routinely $20,30,40$, or 50 years ago.

The decline in physical performance with age has also been demonstrated in a variety of laboratory studies. In fact, there is considerable evidence that muscle strength typically declines from middle age onward (e.g., Bassey \& Harries, 1993; Frontera et al., 2000) and that the age-related decline in strength is beyond that expected on the basis of a reduction in muscle mass (e.g., Amara et al., 2003; Kallman, Plato, \& Tobin, 1990). Such age-related differences in strength and power occur for both upper- and lowerbody muscles (e.g., Candow \& Chilibeck, 2005; Petrella, Kim, Tuggle, Hall, \& Banman, 2005). The effects of those changes can be seen in the comparison of simple manual aiming movements: Older adults typically demonstrate longer movements times than do younger adults (e.g., Lyons, Elliott, Swanson, \& Chua, 1996). However, there may be an exception to that general rule, and that exception involves humans' ability to produce the rapid aimed eye movements known as saccades.

People use saccadic eye movements to quickly orient the focus of gaze, the fovea, to specific locations in their visual field. The fovea is the tiny portion of the retina that contains the vast majority of the cone cells needed to convey detailed visual information to the brain. Thus, to successfully interact with their environment, they need to constantly move the fovea to various portions of the visual field throughout

Correspondence address: Jay Pratt, Department of Psychology, University of Toronto, 100 George Street, Toronto, ONT M5S 3G3, Canada.E-mail address: pratt@psych.utoronto.ca 
each day. Indeed, it is generally estimated that people produce three or four saccades for each second that they are awake (Henderson, Williams, Castelhano, \& Falk, 2003). Given the important and ubiquitous nature of saccades, it is not surprising that researchers have directed a considerable amount of attention to understanding how such movements are planned and produced. Their research has revealed a number of interesting aspects of saccadic movements. Trottier and Pratt (2005), for example, found that saccades are initiated more quickly and reach greater magnitudes of velocity and acceleration than are any other overt movements. Moreover, unlike other movements, saccades conform to a strict linear relationship between movement time and amplitude, a pattern known as the main sequence. The relative simplicity of saccadic neural circuitry, coupled with various anatomical advantages (low inertia, high accuracy), makes the saccadic system ideal to study for both motorcontrol and motor-programming researchers.

Despite the voluminous literature on saccadic function, very few researchers have examined issues regarding the effects of aging on the saccadic eye-movement system. Moreover, the researchers' focus in most of those studies was on issues related to the planning, or programming, of saccades, such as the comparison of changes in the error rates of antisaccades in younger and older adults. The general finding from such studies is that older adults have longer saccadic reaction times (SRTs; e.g., Yang \& Kapoula, in press; Yang et al., 2006) and make more directional antisaccade errors (i.e., they erroneously move their eyes more often to the target location) than do younger adults (e.g., Bojko, Kramer, \& Peterson, 2004; Nieuwenhuis, Ridderinkhof, de Jong, Kok, \& van der Molen, 2000). Although the researchers who conducted the aforementioned motorprogramming studies included measurements of movement time (MT) and amplitude, they did not include more detailed descriptions of the movements because that information was not germane to the questions they had posed. In fact, a handful of researchers have examined age-related differences in peak velocity, and those studies have produced a discordant variety of findings: Spooner, Sakala, and Baloh (1980), Abel, Troost, and Dell'Osso (1983), Moschner and Baloh (1994), and Sharpe and Zackon (1987) reported lower peak velocities in older adults, whereas Munoz, Broughton, Goldring, and Armstrong (1998), Shafiq-Antonacci et al. (1999), and Warabi, Noda, and Kato (1986) reported no differences. There are a few possible factors that can account for those discrepancies across studies. First, none of researchers explicitly designed those studies so that they could examine motor-control processes, and peak velocity was not a central variable for their research topics. Second, it is difficult to get accurate measurements beyond MT and amplitude. The difficulty arises because saccadic MTs are very short (ranging from $20-80 \mathrm{~ms}$ ), and acquiring enough samples to obtain accurate Velocity $\times$ Time profiles requires eye-tracking equipment with sampling frequencies above $200 \mathrm{~Hz}$ (temporal resolution of less than $5 \mathrm{~ms}$ ). The equip- ment that researchers used in the previously noted studies performed below that critical frequency, whereas the equipment we used in the current study sampled at $500 \mathrm{~Hz}$. Third, target amplitudes varied considerably across the studies (ranging from $5^{\circ}$ to $20^{\circ}$ ), and it may be more difficult to detect differences in peak velocity with shorter amplitude movements.

Unlike the aforementioned researchers, Abrams, Pratt, and Chasteen (1998) examined very detailed aspects of saccades to understand what changes in oculomotor control processes, if any, occur with advancing age. Their basic paradigm was quite simple: Participants fixated on a central cross while planning a saccade to a peripheral target either $3.5^{\circ}, 5.5^{\circ}$, or $7.5^{\circ}$ to the right. Following an auditory countdown and go tone, younger and older adults looked, as quickly as possible, to the peripheral target. The key to the study was that high-speed temporal sampling allowed for the derivation of accurate instantaneous velocity and acceleration profiles, providing important kinematic landmarks of peak acceleration, peak velocity, and peak deceleration. Most interesting, Abrams et al. found no age-related differences in any of their measurements of saccadic function: MT, amplitude, peak velocity, peak acceleration, and peak deceleration. The acceleration profiles provided especially insightful information because acceleration values approximated the value of the force being applied to the eye (because force $=$ mass $\times$ acceleration, and the mass of the eye presumably remained roughly constant for each participant throughout each testing session). Thus, the shape of the Acceleration $\times$ Time profiles essentially revealed the shape of the force pulse used to produce the saccades, and those profiles also revealed no age-related differences.

Abrams et al.'s (1998) finding of no age-related differences in the production of saccades contrasts starkly with the general idea that as people age, they move more slowly and less forcefully. Indeed, those researchers noted that the saccadic motor system may be singular among motor systems by being relatively impervious to aging. However, Abrams and colleagues examined a very limited set of saccadic eye movements, all under $10^{\circ}$, all in the same direction, and all programmed well in advance of the go signal. Whereas there may have been no age-related differences within the confines of their study, the task used is not especially representative of the breadth of saccadic function. For example, volitionally initiated saccades are characterized by more activation in the frontal and supplementary eye fields, whereas activation in the parietal eye fields tends to be associated with reflexively initiated saccades. In addition, horizontally directed saccades rely on phasic (burst) neurons in the paramedian pontine reticular formation and tonic neurons in the prepositus hypoglossi, whereas vertically directed saccades rely on phasic neurons in the interstitial nucleus of the medial longitudinal fasciculus and tonic neurons in the interstitial nucleus. Finally, in saccades under $10^{\circ}$, the oculomotor system tends to use force modulation to control amplitude, whereas in saccades over 
$10^{\circ}$, it tends to use the temporal modulation of maximum force for such control. Thus, there are a variety of reasons to suspect that conclusions reached by Abrams et al. may not have captured the full extent of saccadic function.

Our purpose in the present study was, like that of Abrams et al. (1998), to use detailed kinematic measurements of saccadic eye movements to gain some insights into possible age-related changes in oculomotor control. Expanding on the method used by Abrams et al., in the present study we examined (a) volitional and reflexive saccades, (b) horizontal and vertical saccades, and (c) a number of different saccadic amplitudes. If Abrams et al. are correct in their assertion that the saccadic motor system is relatively impervious to aging, then we should find no significant agerelated differences in the key variables of MT, amplitude, peak velocity, peak acceleration, and peak deceleration across any of the experimental manipulations. The finding of age-related differences among those variables would indicate that certain parts of the saccadic motor system are, in fact, sensitive to aging. Furthermore, knowing which variables, under which conditions, are different in older adults will help to pinpoint the underlying neural structures or processes that are sensitive to the effects of aging.

\section{EXPERIMENT 1}

Following the method of Abrams et al. (1998), in the first experiment of the present study we examined the kinematic features of volitionally generated eye movements. Unlike Abrams et al., in the present study we used a wider range of target amplitudes $\left(4^{\circ}, 8^{\circ}\right.$, and $12^{\circ}$ ) and directions (up, down, left, right) to obtain a broader understanding of aging and saccades.

\section{METHOD}

\section{Participants}

Ten younger $($ mean age $=19.3$ years, range $=18-21$ years; mean education $=14.1$ years) and 10 older (mean age $=66.7$ years, range $=64-70$ years; mean education $=15.4$ years) adults participated in the study. The younger adults were undergraduate psychology students at the University of Toronto and received course credit for their participation. We recruited the older adults through the Department of Psychology's Elderly Participant Pool, and they received $\$ 10$ per hour. All of the participants had at least 20/20 vision (with or without corrective lenses) and were naive to our purpose in the experiment.

\section{Apparatus}

The eye tracker used in the current study was the SR Research Ltd. (Osgoode, ONT, Canada) EyeLink system. That system has high spatial resolution $\left(0.005^{\circ}\right)$ and a sampling rate of $500 \mathrm{~Hz}$ (2-ms temporal resolution). The EyeLink headband has three cameras, allowing simultaneous tracking of both eyes and head position for head-motion compensation. We monitored both of the participant's eyes; we used the conjugate signal ([left eye + right eye]/2) for the analyses. Saccade movement onset was detected when acceleration $\left(8,000^{\circ} / \mathrm{s}^{2}\right)$ and velocity $\left(30^{\circ} / \mathrm{s}\right)$ thresholds were reached with saccades of $0.5^{\circ}$ of visual angle or greater, and movement offset was detected when velocity fell below $30 \%$ s and remained so for 10 consecutive samples. Trials in which blinks or anticipatory saccades occurred were rerun at the end of the session.

Stimulus displays were presented on two monitors, one for the participant (a 19-in. Dell monitor with a refresh rate of $120 \mathrm{~Hz}$ and a screen resolution of 800-600 pixels) and the other for the experimenter (one of the authors). The experimenter used the monitor to obtain feedback in real time about the participant's computed gaze position. The feedback allowed the experimenter to evaluate system accuracy and to initiate a recalibration if necessary. In general, the average error in the computation of gaze position was less than $0.5^{\circ}$.

We performed a 9-point calibration procedure at the beginning of the experiment, followed by a 9-point calibration accuracy test. We repeated calibration if any point was in error by more than $1^{\circ}$ or if the average error for all points was greater than $0.5^{\circ}$.

\section{Procedure}

Each trial began with a display that consisted of a white central fixation dot $\left(0.5^{\circ}\right)$ and a white peripheral target dot $\left(0.75^{\circ}\right)$ on a black computer screen. After the experimenter ensured that the participant was correctly fixating on the fixation dot, there was a 1,000-ms delay and then a centrally presented auditory go signal. We instructed the participants to look to the peripheral target as quickly as possible upon presentation of the go signal. The peripheral target was equally likely to appear above, below, left, or right of the fixation dot, and was equally likely to be $4^{\circ}, 8^{\circ}$, or $12^{\circ}$ from the fixation dot. Because of the constraints of the testing room, $12^{\circ}$ was as distant a target as possible in all four directions. Once each saccade was finished, the screen was cleared, and the experimenter pressed a manual button press to initiate the next trial. Each participant completed 360 trials (30 trials per direction per amplitude), and breaks were provided every 30-40 trials.

\section{RESULTS AND DISCUSSION}

A preliminary analysis revealed no differences between left and right or up and down saccades across all variables; we therefore collapsed the data into horizontal and vertical saccade conditions. Thus, we analyzed all of the variables with a 2 (target dimension: vertical or horizontal) $\times 3$ (target amplitude: $4^{\circ}, 8^{\circ}$, or $\left.12^{\circ}\right) \times 2$ (age: younger or older) mixed analysis of variance (ANOVA).

\section{Basic Saccade Features}

The means of the basic saccade features (SRT, MT, and amplitude) are shown in the top portion of Table 1 (with corresponding standard deviation values in the top portion 
of Table 2). Although we found no main effect of target dimension on SRT, $F(1,18)<1$, vertical saccades had greater MTs than did horizontal saccades, $F(1,18)=21.9$, $p<.001$, and vertical saccades traveled farther than horizontal saccades did, $F(1,18)=414, p<.001$. Likewise, we found no main effect of target amplitude on SRT, $F(2,36)$ $<1$, whereas saccadic MT, $F(2,36)=307, p<.001$, and saccadic amplitude, $F(2,36)=10,735, p<.001$, increased with target amplitude. We found the same pattern of effects for the Target Dimension $\times$ Target Amplitude interaction; we found no effects on SRTs, $F(1,18)<1$, but did find significant interactions for MT, $F(1,18)=6.5, p<.004$, and saccade amplitude, $F(1,18)=20.6, p<.001$.

Focusing on the topic of the current study, we found no main effect of age on SRT, MT, or saccade amplitude, $F \mathrm{~s}(1$, $18)<1$. In addition, we found no Age $\times$ Target Dimension interactions for SRT, MT, and saccade amplitude, $F \mathrm{~s}(1$, $18)<1$. Furthermore, we found no Age $\times$ Target amplitude interactions for SRT, $F(2,36)<1$, MT, $F(1,18)<1.4, p>$ .25 , and saccade amplitude, $F(2,36)<1.3, p>.13$. Finally, we found no Age $\times$ Target Dimension $\times$ Target Amplitude interactions for any of the variables, all $F \mathrm{~s}(2,36)<1$.

As one would expect, saccades to farther targets traveled greater distances and took more time than did saccades to closer targets. However, we found considerable differences between horizontal and vertical saccades: Vertical saccades showed considerably more overshoot than did horizontal saccades. The major finding from those variables, replicating the work of Abrams et al. (1998), was that we observed no agerelated differences on MT and saccade amplitude. Moreover, both age groups showed the classic main sequence pattern of a linear relationship between MT and saccade amplitude for both horizontal (younger $r^{2}=.999$; older $r^{2}=.990$ ) and vertical (younger $r^{2}=.991$; older $r^{2}=.995$ ) saccades. Also replicating Abrams et al. was the absence of an age effect on SRT - a finding that is contrary to the results of the bulk of aging studies in which researchers have measured saccadic or manual responses times. The absence of an effect here, and in Abrams et al., could have resulted from the fact that the participants were able to fully program each saccade in advance of the go signal.

\section{Kinematic Saccade Features}

The means of the kinematic features (peak velocity, peak acceleration, and peak deceleration) are shown in Figure 1. The different MT and saccade amplitudes made to vertical and horizontal targets should produce corresponding effects in the kinematic features of the saccades. That was indeed the case because vertical saccades had higher values for peak velocity, peak acceleration, and peak deceleration, $F_{\mathrm{s}}(1,18)$

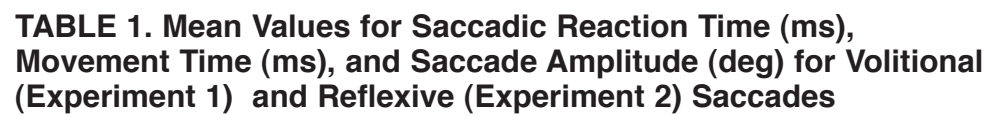

\begin{tabular}{|c|c|c|c|c|c|c|}
\hline \multirow[b]{2}{*}{ Variable } & \multicolumn{3}{|c|}{ Younger } & \multicolumn{3}{|c|}{ Older } \\
\hline & $4^{\circ}$ & $8^{\circ}$ & $12^{\circ}$ & $4^{\circ}$ & $8^{\circ}$ & $\overline{12^{\circ}}$ \\
\hline \multicolumn{7}{|c|}{ Volitional saccades } \\
\hline \multicolumn{7}{|c|}{ SRT } \\
\hline Horizontal & 278 & 277 & 272 & 296 & 288 & 293 \\
\hline Vertical & 272 & 276 & 277 & 298 & 283 & 300 \\
\hline \multicolumn{7}{|c|}{ MT } \\
\hline Horizontal & 47.2 & 58.3 & 69.4 & 45.3 & 54.9 & 64.7 \\
\hline Vertical & 49.9 & 66.2 & 76.8 & 48.4 & 61.6 & 72.3 \\
\hline \multicolumn{7}{|c|}{ Amplitude } \\
\hline Horizontal & 3.45 & 6.87 & 10.14 & 3.64 & 6.82 & 10.11 \\
\hline Vertical & 5.17 & 9.87 & 14.20 & 4.99 & 9.28 & 13.28 \\
\hline \multicolumn{7}{|c|}{ Reflexive saccades } \\
\hline \multicolumn{7}{|c|}{ SRT } \\
\hline Horizontal & 203 & 194 & 195 & 236 & 231 & 244 \\
\hline Vertical & 208 & 206 & 214 & 258 & 261 & 280 \\
\hline \multicolumn{7}{|c|}{ MT } \\
\hline Horizontal & 39.3 & 50.8 & 59.5 & 40.9 & 51.4 & 60.2 \\
\hline Vertical & 46.7 & 59.9 & 69.8 & 46.4 & 58.3 & 69.3 \\
\hline \multicolumn{7}{|c|}{ Amplitude } \\
\hline Horizontal & 3.55 & 6.94 & 10.19 & 3.41 & 6.53 & 9.65 \\
\hline Vertical & 5.49 & 10.59 & 15.27 & 5.14 & 9.87 & 14.45 \\
\hline
\end{tabular}

Note. The values $4^{\circ}, 8^{\circ}$, and $12^{\circ}$ represent the distance of the peripheral target from the fixation dot. 
TABLE 2. Standard Deviation Values for SRT (ms), MT (ms), and Saccade Amplitude (deg) for Volitional (Experiment 1) and Reflexive (Experiment 2) Saccades

\begin{tabular}{|c|c|c|c|c|c|c|}
\hline \multirow[b]{2}{*}{ Variable } & \multicolumn{3}{|c|}{ Younger } & \multicolumn{3}{|c|}{ Older } \\
\hline & $4^{\circ}$ & $8^{\circ}$ & $12^{\circ}$ & $4^{0}$ & $8^{\circ}$ & $\overline{12^{\circ}}$ \\
\hline \multicolumn{7}{|c|}{ Volitional saccades } \\
\hline \multicolumn{7}{|c|}{ SRT } \\
\hline Horizontal & 63.5 & 66.1 & 63.1 & 37.8 & 39.2 & 45.8 \\
\hline Vertical & 62.1 & 71.8 & 79.2 & 45.0 & 53.8 & 58.5 \\
\hline \multicolumn{7}{|c|}{ MT } \\
\hline Horizontal & 15.1 & 11.1 & 12.6 & 4.5 & 5.1 & 7.3 \\
\hline Vertical & 12.1 & 15.1 & 15.9 & 5.7 & 7.8 & 9.6 \\
\hline \multicolumn{7}{|c|}{ Amplitude } \\
\hline Horizontal & 0.42 & 0.75 & 0.95 & 0.57 & 1.00 & 1.48 \\
\hline Vertical & 1.24 & 2.12 & 2.96 & 0.84 & 1.48 & 2.11 \\
\hline \multicolumn{7}{|c|}{ Reflexive saccades } \\
\hline \multicolumn{7}{|c|}{ SRT } \\
\hline Horizontal & 28.5 & 27.8 & 28.7 & 28.5 & 34.2 & 30.6 \\
\hline Vertical & 30.9 & 25.8 & 27.5 & 31.0 & 35.7 & 41.3 \\
\hline \multicolumn{7}{|c|}{ MT } \\
\hline Horizontal & 3.6 & 3.2 & 3.9 & 3.7 & 3.3 & 4.6 \\
\hline Vertical & 9.5 & 10.5 & 8.0 & 5.8 & 6.1 & 7.8 \\
\hline \multicolumn{7}{|c|}{ Amplitude } \\
\hline Horizontal & 0.38 & 0.79 & 1.14 & 0.41 & 0.72 & 0.94 \\
\hline Vertical & 0.73 & 1.08 & 1.50 & 0.83 & 1.70 & 2.71 \\
\hline
\end{tabular}

Note. $4^{\circ}, 8^{\circ}$, and $12^{\circ}$ represent the distance of the fixation dot from the peripheral target. $\mathrm{SRT}=$ saccadic reaction time. $\mathrm{MT}=$ movement time.

$=16.2,23.6$, and 1,571, respectively, all $p \mathrm{~s}<.001$. As one would expect, we found greater magnitudes of peak velocity, peak acceleration, and peak deceleration, $F \mathrm{~s}(2,36)=368$, 180 , and 968, all $p \mathrm{~s}<.001$, as target amplitude increased. We found similar patterns of data for the interaction of Target Dimension $\times$ Target Amplitude on peak velocity, $F(2,36)=$ $11.5, p<.001$, and peak acceleration, $F(2,36)=6.7, p<.003$, but not for peak deceleration, $F(2,36)<2.9, p<.10$.

Following the basic saccade-feature data, we found no main effects of age for peak velocity, $F(1,18)<1.8, p>.20$, peak acceleration, $F(1,18)<1.3, p>.28$, and peak deceleration, $F(1,18)<1$. It should be noted, however, that in all cases the older adults showed higher absolute magnitudes of those kinematic variables than did the younger adults (see Figure 1). We found no Age $\times$ Target Dimension, Age $\times$ Target Amplitude, or Age $\times$ Target Dimension $\times$ Target Amplitude interactions (all $F \mathrm{~s}<1$ ).

We also examined the shape of the force-time curves produced by the two groups of participants by looking for age effects on the time and position at which peak velocity, peak acceleration, and peak deceleration were reached. As would be expected by the preceding analysis, we found no age-related differences (all $F_{\mathrm{s}}<1$ ), confirming that, as Abrams et al. (1998) first found, older adults produced force pulses to move the eye in much the same way as younger adults did.
One could also use another set of kinematic data to examine whether movements are largely preprogrammed or are capable of being controlled by feedback-based processes. Given the short MTs associated with saccades, it is generally accepted that saccades are ballistic in nature, entirely programmed, and insensitive to modification once initiated. By measuring limb movements, however, Khan, Elliott, Coull, Chua, and Lyons (2002) found that examining the associated variability in the position at which the kinematic landmarks of peak acceleration, peak velocity, peak deceleration, and movement endpoint occur provides a sensitive comparison of ballistic and controlled movements. Evidence for feedback-based control processes is basically obtained if the variability around the timing of those landmarks increases in the earlier stages of the movement (peak acceleration to peak deceleration) and subsequently decreases in the later stages of the movement, especially from peak deceleration to movement endpoint. If no such reduction, or an increase in variability over time, is found, then one can characterize the movements as ballistic. The present study, as far as we know, is the first application of the variability analysis to saccades. As can be seen in the upper panel of Figure 2 , variability in the position at the landmarks did not differ across age groups, and both groups generally showed either no change or an increase from peak deceleration to 


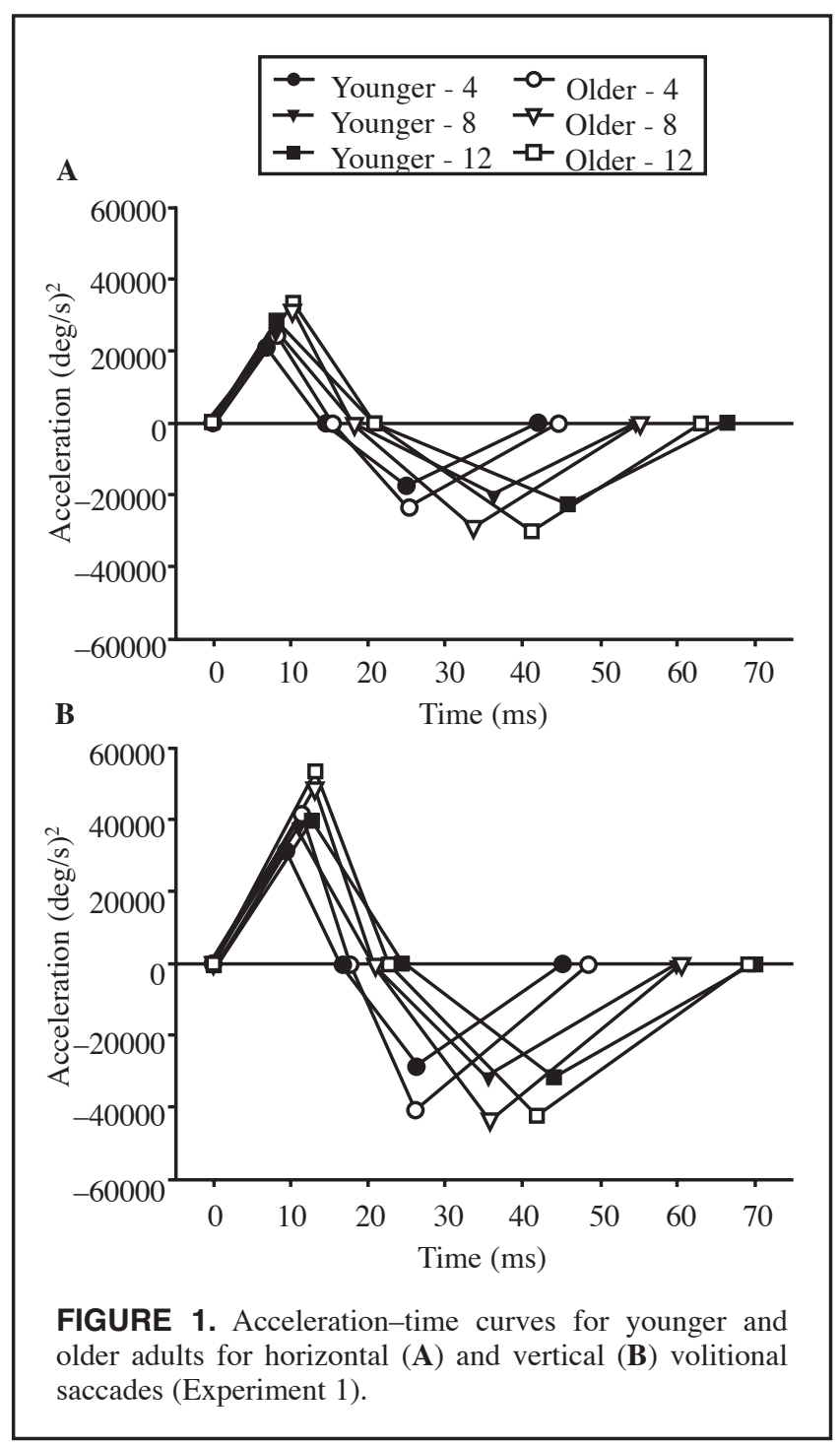

movement endpoint. The longest vertical movements were the exception because those movements may have been the only saccades with sufficiently long durations to allow for some feedback-based adjustments. Thus, both groups produced largely ballistic saccades to close targets but were able to correct their saccades to farther targets while the saccade was being completed.

The kinematic analyses showed that, consistent with Abrams et al. (1998), older adults did not produce saccades that were slower or less forceful than those of younger adults. In fact, the differences between vertical and horizontal saccades were much larger than were the differences between participants whose ages differed by almost 50 years.

\section{EXPERIMENT 2}

As previously mentioned, one possible confounding factor in the previous experiment and in the Abrams et al. (1998) study was that we compared younger and older adults' performance on volitionally initiated saccades. With such saccades, presumably all of the necessary motor pro-

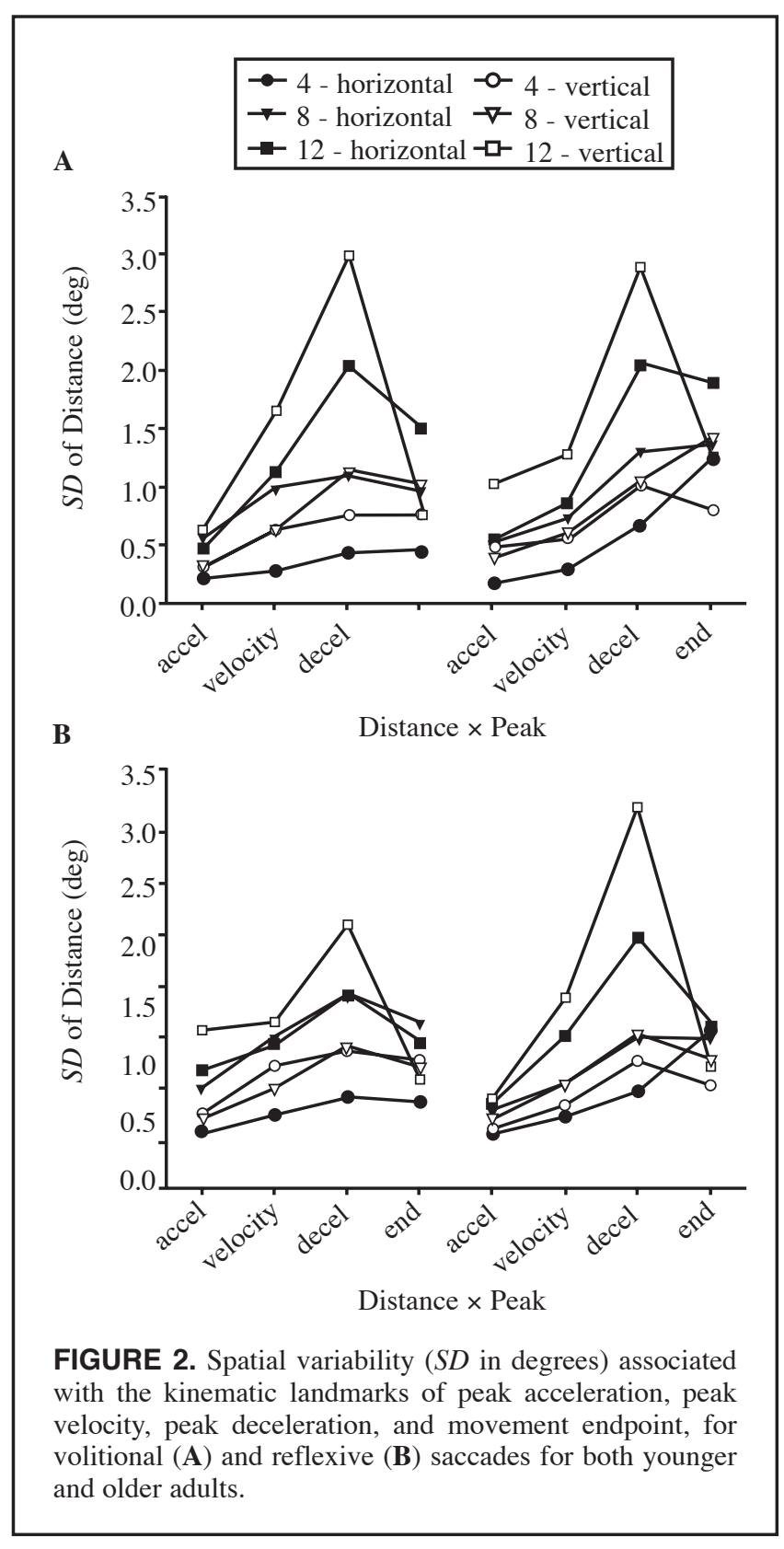

gramming is accomplished well before the go signal, and the extensive preprogramming may greatly reduce, or even eliminate, any possible age-related differences in saccade production. Because of that possibility, in the second experiment we examined reflexive saccades; in that experiment, the onset of the peripheral target also served as the go signal, eliminating the possibility of advance programming.

\section{METHOD}

\section{Participants}

Ten younger $($ mean age $=18.9$ years, range $=18-21$ years; mean education $=14.5$ years $)$ and 10 older (mean age $=66.1$ years, range $=61-72$ years; mean education $=$ 14.9 years) adults participated in the study. The younger 
adults were undergraduate psychology students at the University of Toronto and received course credit for their participation. We recruited the older adults through the Department of Psychology's Elderly Participant Pool, and they received $\$ 10$ per hour. All of the participants had 20/20 vision (with or without corrective lenses) and were naive to our purpose in the experiment. None of the participants had taken part in Experiment 1.

\section{Apparatus and Procedure}

The apparatus used in Experiment 2 was the same as that used in Experiment 1. The procedure for the present experiment was also the same as that used in Experiment 1 (target amplitudes of $4^{\circ}, 8^{\circ}$, and $12^{\circ}$; target directions of up, down, left, and right), except that the initial display consisted of only the central fixation cross. One thousand milliseconds after successful fixation was achieved, a target abruptly appeared at one of the 12 possible locations (with the fixation dot remaining present). Participants were instructed to look to the target as soon as it appeared.

\section{RESULTS AND DISCUSSION}

As before, a preliminary analysis revealed no differences between left and right or up and down saccades across all variables; we therefore collapsed the data into horizontal and vertical saccade conditions. Thus, we analyzed all of the variables with a 2 (target dimension: vertical or horizontal) $\times 3$ (target amplitude: $4^{\circ}, 8^{\circ}$, or $\left.12^{\circ}\right) \times 2$ (age: younger or older) ANOVA.

\section{Basic Saccade Features}

The bottom portion of Table 1 shows the means of the basic saccade features (SRT, MT, and amplitude); corresponding $S D$ values are shown in the bottom portion of Table 2). All three variables showed main effects of target dimension: Vertical saccades had longer SRTs, and longer MTs, and traveled farther, $F \mathrm{~s}(1,18)=31.2,39.2$, and 71.4, respectively, all $p s<.001$, than did horizontal saccades. Likewise, for the main effects of target amplitude, we found the following to be longer with greater target amplitudes: SRTs, $F(2,36) 4.8, p<.02$; MTs, $F(2,36)=991, p<.001$; and saccade amplitudes, $F(2,36)=3,032, p<.001$. That pattern was again repeated for the Target Dimension $\times$ Target Distance interactions; we found significant effects with SRT, MT, and saccade amplitude, $F \mathrm{~s}(1,18)=8.7,10.3$, and 50.9, respectively, all $p s<.001$.

Unlike the previous experiment with volitional saccades, the reflexive saccade task in the present experiment showed a main effect for age on SRT, $F(1,18)=18.1, p<.001$; older adults had longer SRTs than did younger adults. Although we found no age main effect of MT, $F(1,18)<1$, younger adults did have longer saccade amplitudes, $F(1,18)=4.8, p<.04$. The analysis of SRTs also revealed an Age $\times$ Target Dimension interaction for SRT, $F(1,18)=5.7, p<.03$, indicating that SRTs were longer for vertical than for horizontal saccades only for the older adults. We found no such interaction for MT or saccade amplitude, $F \mathrm{~s}(1,18)<1$. We found a trend for an Age $\times$ Target Amplitude interaction for SRT, $F(1,18)$ $=3.2, p<.06$, and for saccade amplitude, $F(1,18)=2.5, p<$ .1 , but not for MT, $F(1,18)<1$. Finally, we found no Age $\times$ Target Dimension $\times$ Target Amplitude interactions for any of the variables, all $F \mathrm{~s}(2,36)<1$.

Once again, saccades to farther targets traveled greater distances and took more time than did saccades to closer targets, and, as we previously found, there were considerable differences between horizontal and vertical saccades. Although older adults made slightly longer saccades than younger adults did, both age groups showed the main sequence pattern for both horizontal and vertical saccades between MT and saccade amplitude (younger $r^{2}=.996$; older $r^{2}=.998$ ) and vertical (younger $r^{2}=.994$; older $r^{2}$ $=.996$ ) saccades. Finally, as expected with speeded tasks in which response selection is required, older adults had slower SRTs than did younger adults.

\section{Kinematic Saccade Features}

Figure 3 shows the means of the kinematic saccade features (peak velocity, peak acceleration, and peak deceleration). As noted earlier, vertical saccades had higher values for peak velocity, peak acceleration, and peak deceleration, respectively, $F \mathrm{~s}(1,18)=31.9,60.9$, and 43.7, all $p s<.001$. Furthermore, we found greater magnitudes of peak velocity, peak acceleration, and peak deceleration, $F_{\mathrm{S}}(2,36)=$ 693, 223, and 39.2, respectively, all $p s<.001$, as target amplitude increased. We found similar patterns of data for the interaction of Target Dimension $\times$ Target Amplitude on peak velocity, $F(2,36)=25.9, p<.001$, and on peak acceleration, $F(2,36)=21.6, p<.001$, but not on peak deceleration, $F(2,36)<1.5, p<.20$.

Consistent with the results of the MT analysis, we found no main effects of age for peak velocity, $F(1,18)<1.8, p>.20$, peak acceleration, $F(1,18)<1.2, p>.29$, and peak deceleration, $F(1,18)<1$. As in the previous experiment, and visible in Figure 3, older adults had higher absolute magnitudes of those kinematic variables than did the younger adults. We found no Age $\times$ Target Dimension (all $F \mathrm{~s}<1$ ), Age $\times$ Target Amplitude (all $F \mathrm{~s}<2.0, p s>.15$ ), or Age $\times$ Target Dimension $\times$ Target Amplitude (all $F \mathrm{~s}<1.5$, ps $>.23$ ) interactions.

As in the previous experiment, we also examined the shape of the acceleration-time curves produced by the two groups of participants by looking for age effects on the time and position at which peak velocity, peak acceleration, and peak deceleration were reached (see the bottom panel of Figure 2). Although we found no age age-related difference regarding the time and position of peak acceleration and peak deceleration (all $F \mathrm{~s}<2.2, p \mathrm{~s}>.15$ ), older adults did reach peak velocity earlier in both time, $F(1,18)=7.8, p<$ .02 , and position, $F(1,18)=4.5, p<.05$, than did younger adults. Most interesting, the shorter amplitude saccades produced by the older adults did not result in the shifting forward of all the force landmarks; rather, only the time and position of peak velocity were affected. In addition, only 


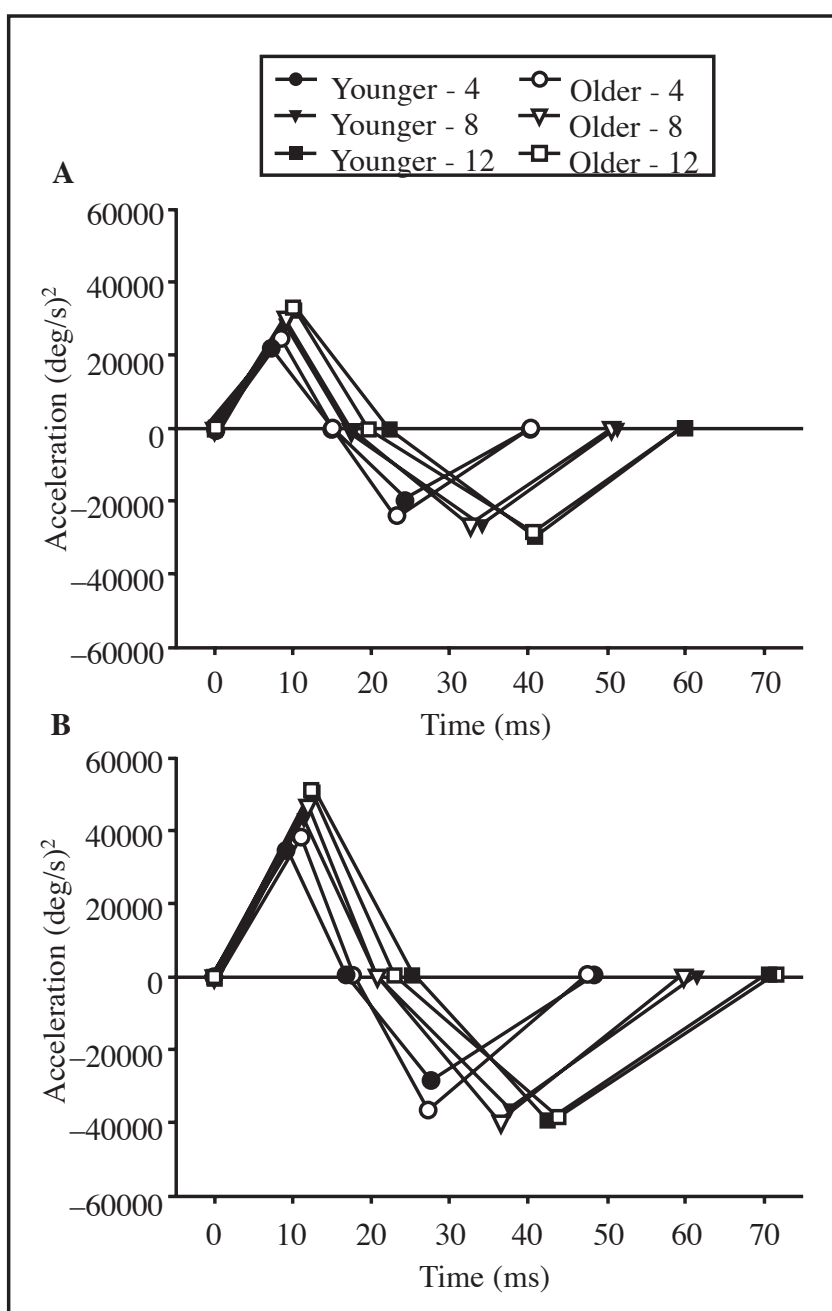

FIGURE 3. Acceleration-time curves for younger and older adults for horizontal (A) and vertical (B) reflexive saccades (Experiment 2).

the longest vertical movements decreased in spatial variability from peak deceleration to movement endpoint (see the bottom panel of Figure 2), but there were no differences between age groups. As with the volitional saccades, the reflexive saccades appeared to be ballistic for younger and older adults, except for long vertical saccades.

Consistent with the earlier experiment and the findings of Abrams et al. (1998), the saccades of older adults were not slower or less forceful than were those of younger adults. Indeed, the older adults produced reflexive saccades that were longer in amplitude but not longer in MT. Thus, although not statistically different, the magnitude of speed and force values for the older adults was slightly greater than those we found for the younger adults. Once again, those differences were diminutive compared with the differences we found between horizontal and vertical saccades.

\section{GENERAL DISCUSSION}

The main finding from the two experiments in the present study is that the saccadic motor system did not show the effects of aging that are often observed in the other skeletal motor systems. The older adults were able to reach the same velocities and produce the same forces as were participants 40 to 50 years younger than they were. Indeed, the differences between how horizontal and vertical saccades were produced were far greater than anything found between the two age groups. The lone exception was SRT with reflexive saccades; in that case, older adults needed more time to initiate movements. However, the difference in SRT disappeared with volitional saccades when sufficient programming time was provided before the saccade was elicited. It is worth noting here that our elderly sample, with an average age of approximately 66 years, would be considered a younger elderly sample, and their relative youth may have also contributed to the absence of the SRT effect found in Experiment 1. In addition, older adults may not show a slowing of SRTs in some conditions, such as short amplitude movements, with the removal of the fixation point (Yang et al., 2006).

With evidence that there is little decline in saccade performance with age, the important, but difficult to answer, question becomes: Why is the saccadic motor system, unlike the other motor systems, able to maintain speed and force across the life span? Two broad classes of answers are possible, one focusing on the unique structure of the saccadic motor system, the other focusing on the unique performance of the system. Turning first to the latter possibility, saccades may not show declines in velocity or acceleration because constant saccadic activity keeps that system more fit than other systems. No other motor system in the human body approaches the nearly 200,000 movements that the saccadic motor system makes each day of a person's life. Such a high and constant level of activity may simply keep the saccadic motor system more fit than other systems. Indeed, presumably even sedentary individuals still produce about the same number of saccades on a daily basis as do very physically active people.

The other answer for why the saccadic system does not show a decline with aging is that saccades have physical structure that is unlike those in all other motor systems. One produces saccades by using three pairs of muscles to move the eye in the orbit. Unlike limb movements, saccades are produced only by agonist activity; that activity is specified by the ratio of forces between the two muscles making up each pair. The agonist activity rapidly moves the eye to the desired location without subsequent activation of the antagonist muscle to break or facilitate corrections in saccadic trajectory online. Although the antagonist activity that typifies limb movements is not seen with saccades, it is not clear what attributes of the saccade motor system would produce a resistance to the effects of aging. Perhaps the system is "overengineered," such that the pairs of muscles are capable of moving the relatively light eye at even greater forces but are limited by some neural control mechanism. Consistent with that notion, there is evidence that the extraocular muscles that move the eyes do show progressive morphological 
changes with aging, such as loss of myofibrils (contractile elements in the muscle fibers; McKelvie, Friling, Davey, \& Kowal, 1999) and degeneration of mitochondria (cellular organelles that provide energy to the muscle fibers; Miller, 1975). Those changes are similar to the age-related changes found in limb muscles (e.g., Cooper, Mann, \& Schapira, 1992). Thus, it appears that the performance of the saccadic system is relatively preserved despite typical age-related declines in contractile tissue.

A second alternative, in which the structure of the saccadic system allows for more age-resistant motor control processes, is also possible. Here, the simple structure of the oculomotor system may facilitate planning and control strategies that are less susceptible to the effects of aging. Indeed, it has been demonstrated that older adults tend to show greater deficits in complex, coordinated limb movements (e.g., Barry, Riek, \& Carson, 2005) than do younger adults. Moreover, research on the age-related decline in the speed and accuracy of rapid aiming movements suggests that the main difference between younger and older people is that older participants cannot use their online control systems as efficiently as can their younger counterparts. For example, Welsh and Elliott (2006) found that the longer movement times exhibited by older participants resulted from their spending a longer period of time after peak velocity, a stage in which correction processes are activated. Most interesting, there were no reliable differences in the number of discrete corrections or variability in the locations of the kinematic markers, suggesting that the two groups went through the same correction processes, only that the younger participants completed them more efficiently (see also Chaput \& Proteau, 1996; Lyons et al., 1996). In the context of the present experiments, the reason that we observed no differences in saccadic eye movements could be that there is no age-related decline in the ability to program and control saccadic eye movement. That is, because eye movements, at least those of moderate amplitude, are ballistic in nature and are completed with minimal influence from online corrective processes (our analysis of the variability of the position of the kinematic markers for these saccades supports that idea), there is no reason for those types of actions to deteriorate with age. On the other hand, the decrease in variability of spatial position from peak deceleration to the end of the movement for the $12^{\circ}$ saccades suggests that online control processes may influence saccades of greater amplitude. Although the nature of that online control is uncertain, the fact that there were no agerelated differences in this variability analysis reveals that, even when online control may be involved, the absence of age-related differences is something that is unique to the production of saccades.

Although the unique-structure answer provides a plausible reason why saccades are largely unaffected by aging, the reality of the situation is that not enough is known about aging and oculomotor system to allow researchers to make any conclusive statements. Moreover, the sac- cadic system's resistance to aging does not appear to be a general property of the oculomotor system because smooth pursuit movements show age-related increases in latencies (Knox, Davidson, \& Anderson, 2005) and decreases in peak velocities (Morrow \& Sharpe, 1993). Perhaps the best way to proceed in answering the question of why saccades are resistant to aging may be to use more invasive measures, such as directly comparing the oculomotor musculature of younger and older participants, both nonhuman and human (something which, as far as we know, has never been done). Regardless of the underlying cause, it is at least comforting to know that as people generally get slower and weaker with age, at least one motor system in their bodies acts forever young - the saccadic motor system.

\section{ACKNOWLEDGMENTS}

Grants from the Canadian Institutes of Health Research (CHIR), the Natural Sciences and Engineering Council, and a Premier's Research Excellence Award (PREA), to Jay Pratt supported this work. Michael Dodd received support from a William George Scott Fellowship in Gerontology and also from the CIHR, and Timothy Welsh received support from PREA. We thank Leo Trottier and Jen Boyd for their help with programming and data collection.

\section{REFERENCES}

Abel, L. A., Troost, B. T., \& Dell'Osso, L. F. (1983). The effects of age on normal saccadic characteristics and their variability. Vision Research, 23, 33-37.

Abrams, R. A., Pratt, J., \& Chasteen, A. L. (1998). Aging and movement: Variability of force pulses for saccadic eye movements. Psychology and Aging, 13, 387-395.

Amara, C. E., Rice, C. L., Koval, J. J., Paterson, D. H., Winer, E. M., \& Cunningham, D. A. (2003). Allometric scaling of strength in an independently living population age 55-86 years. American Journal of Human Biology, 15, 48-60.

Barry, B. K., Riek, S., \& Carson, R. G. (2005). Muscle coordination during rapid force production by young and older adults. Journal of Gerontology: Medical Sciences, 60A, M232-M240.

Bassey, E. J., \& Harries, U. J. (1993). Normal values for handgrip strength in 920 men and women aged over 65 years, and longitudinal changes over 4 years in 620 survivors. Clinical Science, 84, 331-337.

Bojko, A., Kramer, A. F., \& Peterson, M. S. (2004). Age equivalence in switch costs for prosaccade and antisaccade tasks. Psychology and Aging, 19, 226-234.

Candow, D. G., \& Chilibeck, P. D. (2005). Differences in size, strength, and power of upper and lower body muscle groups in young and older men. Journal of Gerontology: Medical Sciences, 60A, M148-M156.

Chaput, S., \& Proteau, L. (1996). Aging and motor control. Journal of Gerontology: Psychological Sciences and Social Sciences, 51B, P346-P355.

Cooper, J. M., Mann, V. M., \& Schapira, A. H. V. (1992). Analysis of mitochondrial respiratory chain function and mitochond-rial DNA delection in human skeletal muscle: Effect of aging. Journal of the Neurological Sciences, 113, 91-98.

Frontera, W. R., Hughes, V. A., Fielding, R. A., Fiatarone, M. A., Evans, W. J., \& Roubenoff, R. (2000). Aging of skeletal muscle: A 12-yr longitudinal study. Journal of Applied Physiology, 88, $1321-1326$.

Henderson, J. M., Williams, C. C., Castelhano, M. S., \& Falk, R. J. (2003). Eye movements and picture processing during recognition. Perception \& Psychophysics, 65, 725-734. 
Kallman, D. A., Plato, C. C., \& Tobin, J. D. (1990). The role of muscle loss in the age-related decline of grip strength: Crosssectional and longitudinal perspectives. Journals of Gerontology: Medical Sciences, 45, M82-M88.

Khan, M. A., Elliott, D., Coull, J., Chua, R., \& Lyons, J. (2002). Optimal control strategies under different feedback schedules: Kinematic evidence. Journal of Motor Behavior, 34, 45-57.

Knox, P. C., Davidson, J. H., \& Anderson, D. (2005). Age-related changes in smooth pursuit initiation. Experimental Brain Research, 165, 1-7.

Lyons, J., Elliott, D., Swanson, L. R., \& Chua, R. (1996). The use of vision in manual aiming by young and older adults. Journal of Aging and Physical Activity, 4, 165-178.

McKelvie, P., Friling, R., Davey, K., \& Kowal, L. (1999). Changes as a result of ageing in extraocular muscles: A post-mortem study. Australian and New Zealand Journal of Ophthalmology, 27, 420-425.

Miller, J. E. (1975). Aging changes in extraocular muscles. In G. Lennerstrand \& P. Bach-y-rita (Eds.), Basic mechanisms of ocular motility and clinical implications. Oxford, England: Pergamon Press.

Morrow, M. J., \& Sharpe, J. A. (1993). Smooth pursuit initiation in young and elderly subjects. Vision Research, 33, 203-210.

Moschner, C., \& Baloh, R. W. (1994). Age-related changes in visual tracking. Journals of Gerontology: Medical Sciences, 49, M235-M238.

Munoz, D. P., Broughton, J. R., Goldring, J. E., \& Armstrong, I. T. (1998). Age-related performance of human subjects on saccadic eye movement tasks. Experimental Brain Research, 121, 391-400.

Nieuwenhuis, S., Ridderinkhof K. R., de Jong, R., Kok, A., \& van der Molen, M. W. (2000). Inhibitory inefficiency and failures of intention activation: Age-related decline in the control of saccadic eye movements. Psychology and Aging, 15, 635-647.

Petrella, J. K., Kim, J., Tuggle, S. C., Hall, S. R., \& Banman, M. M. (2005). Age differences in knee extension power, contractile velocity, and fatigability. Journal of Applied Physiology, 98, 211-220.

Shafiq-Antonacci, R., Maruff, P., Whyte, S., Tyler, P., Dudgeon, P., \& Currie, J. (1999). The effects of age and mood on saccadic function in older individuals. Journal of Gerontology: Psychological Sciences, 54B, P361-P368.

Sharpe, J. A., \& Zackon, D. H. (1987). Senescent saccades: Effects of aging on their accuracy, latency and velocity. Acta Oto-Laryngologica, 104(5-6), 422-428.

Spooner, J. W., Sakala, S. M., \& Baloh, R. W. (1980). Effect of aging on eye tracking. Archives of Neurology, 37, 575-576.

Trottier, L., \& Pratt, J. (2005). Visual processing of targets can reduce saccadic latencies. Visual Research, 45, 1349-1354.

Warabi, T., Noda, H., \& Kato, T. (1986). Effect of aging on sensorimotor functions of eye and hand movements. Experimental Neurology, 92, 686-697.

Welsh, T. N., \& Elliott, D. (2006). Aging and speed-accuracy tradeoffs in rapid aiming movements. Manuscript in preparation.

Yang, Q., \& Kapoula, Z. (in press). The control of vertical saccades in aged subjects. Experimental Brain Research.

Yang, Q., Kapoula, Z., Debay, E., Coubard, O., Orssaud, C., \& Samson, M. (2006). Prolongation of latency of horizontal saccades in elderly is distance and task specific. Vision Research, 46, 751-759.

Submitted December 12, 2005

Revised March 22, 2006 
Copyright of Journal of Motor Behavior is the property of Heldref Publications and its content may not be copied or emailed to multiple sites or posted to a listserv without the copyright holder's express written permission. However, users may print, download, or email articles for individual use. 